\title{
INTERVENTIONAL CARDIOLOGY AND SURGERY
}

\section{Simvastatin attenuates leucocyte-endothelial interactions after coronary revascularisation with cardiopulmonary bypass}

\author{
M Chello, P Mastroroberto, G Patti, A D'Ambrosio, M Cortez Morichetti, G Di Sciascio, \\ E Covino
}

Heart 2003;89:538-543

See end of article for authors' affiliations

Correspondence to

Dr Massimo Chello

Department of Cardiac

Surgery, University Campus

Bio Medico di Roma, Via E

Longoni 83, Rome 00155,

Italy;

m.chello@unicampus.it

Accepted

9 January 2003

\begin{abstract}
Objective: To investigate the effects of preoperative simvastatin treatment on leucocyte-endothelial interactions following coronary artery bypass surgery with cardiopulmonary bypass.

Design: Double blind crossover study. Experiments on polymorphonuclear cells (neutrophils) were done at the end of cardiopulmonary bypass and one hour postoperatively. Endothelial P-selectin expression and neutrophil/endothelial adhesion were evaluated under either normoxic or hypoxic conditions.

Setting: University hospital (tertiary referral centre).

Patients: Three groups of patients undergoing coronary bypass surgery: 20 patients taking simvastatin for cholesterol control, 16 patients not responsive to simvastatin, and 20 controls.

Main outcome measures: Expression of neutrophil CD1 1 b and endothelial P-selectin; adhesion of neutrophils to endothelium.

Results: Cardiopulmonary bypass resulted in a significant increase in neutrophil CD 11 b expression in all groups. Similarly, the exposure of saphenous vein to hypoxia/reoxygenation induced an augmentation of endothelial P-selectin. However, both neutrophil CD $11 \mathrm{~b}$ expression and endothelial P-selectin exocytosis were less in the simvastatin groups than in the controls. Cardiopulmonary bypass and controlled hypoxia/reoxygenation stimulated neutrophil/endothelial adhesion, but the number of adhering cells was less in the simvastatin groups than in the controls, irrespective of the cholesterol concentration. Treatment of endothelial cells with L-NAME completely reversed the effects of simvastatin.

Conclusions: Pretreatment with simvastatin reduces neutrophil adhesion to the venous endothelium in patients undergoing coronary surgery, irrespective of its efficacy at lowering cholesterol concentration.
\end{abstract} and immunoglobulin superfamily members)..$^{12}$ NO has been shown to modulate leucocyte-endothelial cell interactions by suppressing the upregulation of several endothelial cell and neutrophil adhesion molecules, including P-selectin, VCAM-1,

and CD1lb/CD18. ${ }^{11}{ }^{13}$ Thus a reduced bioavailability of NO might cause progression and amplification of tissue injury, with neutrophil infiltration into inflamed tissues and local release of inflammatory mediators.

Besides the constitutive NO normally produced by endothelial cells, cardiopulmonary bypass has been shown to induce the release of an inducible form of NO (iNO), through the inducible enzyme NO synthase. ${ }^{9}$ Although constitutive NO appears to be cytoprotective and its release is impaired after cardiopulmonary bypass, ${ }^{10}$ several reports have highlighted the direct role of iNO in inducing myocardial and lung dysfunction through its negative inotropic effect, or by causing vasodilatation and increasing vascular permeability. ${ }^{14}$

The 3-hydroxy 3-methyl-glutaryl coenzyme-A (HMG-CoA) reductase inhibitors, simply known as "statins", are substances widely employed in the control of hypercholesterolaemia. The major mechanism of statins is the inhibition of cholesterol synthesis in the liver by blockade of HMG-CoA conversion to mevalonate, the rate limiting step in cholesterol biosynthesis. Clinical trials have shown that statins notably reduce cardiovascular morbidity and mortality in subjects with and without established coronary artery disease, and improve cardiovascular outcome after coronary artery bypass grafting. ${ }^{16-18}$ Nevertheless, from these studies it is evident that

Abbreviations: $C A B G$, coronary artery bypass graft surgery; eNOS, endothelial nitric oxide synthase HMG-CoA, 3-hydroxy 3-methyl-glutaryl coenzyme-A; L-NAME, NG-nitro-L-arginine methyl ester; NO, nitric oxide 
not all the clinical benefits of the agents can be explained by their lipid lowering effects, and a NO mediated antiinflammatory action has been hypothesised. ${ }^{19-21}$ Pruefer and colleagues $^{22}$ showed by intravital microscopy that the administration of simvastatin significantly inhibited leucocyte rolling, adherence, and transmigration in a rat model of the acute inflammatory state. This effect was found to be mediated by downregulation of P-selectin expression on endothelial cells, and is consistent with the downregulation of CD18 on stimulated neutrophils.

In the present study we tested the effects of simvastatin, at doses equivalent to those used orally for cholesterol control, on leucocyte-endothelial interactions in vitro during the inflammatory state following coronary artery bypass surgery with cardiopulmonary bypass.

\section{METHODS}

Among patients admitted for elective coronary artery bypass graft surgery $(\mathrm{CABG})$ at the department of cardiac surgery at Catanzaro medical school, 56 who were taking simvastatin for treatment of hypercholesterolaemia were evaluated. Initiation of treatment with a lipid lowering agent was done at the discretion of the patient's primary physician. A total cholesterol concentration $>6.2 \mathrm{mmol} / \mathrm{l}$ (> $240 \mathrm{mg} / \mathrm{dl}$ ) was used to define hypercholesterolaemia. The patients had not taken any other cholesterol lowering drugs for at least a year. Diabetic patients, patients with renal or hepatic impairment, congestive heart failure, active inflammatory or immunomodulatory diseases, or a history of myocardial infarction less than six months previously, and pregnant women were excluded.

On admission, 36 subjects fulfilled the inclusion criteria and were included in the study. Among these patients, statin treatment was effective at lowering the cholesterol concentration in 20 (group A), and failed to produce therapeutic effects in 16 (group B). These patients were compared with 20 age and sex matched controls undergoing elective CABG who were normocholesterolaemic (group C).

\section{Operative procedure}

All patients underwent CABG with cardiopulmonary bypass using standard procedures. The surgical technique has been described in detail previously. ${ }^{23}$ The same standard anaesthesia protocol was used in all patients. After premedication, a Swan-Ganz catheter was positioned in the central pulmonary artery and a radial artery cannula was inserted. Cardioplegic arrest was achieved with ice cold St Thomas' Hospital solution infused into the ascending aorta.

\section{Study protocol}

Blood samples were taken from the radial artery catheter before the induction of anaesthesia, at the end of cardiopulmonary bypass, and 60 minutes postoperatively. The samples were collected in cooled heparinised syringes which were immediately capped and stored in ice until separation and analysis. The study protocol was approved by the ethics committee of Catanzaro medical school. Informed consent was obtained from each patient.

\section{Saphenous vein segment harvesting and culture}

Segments of saphenous vein harvested at the time of bypass surgery were opened carefully and placed endothelial side up in separate $5 \mathrm{ml}$ round cell culture dishes containing $3 \mathrm{ml}$ of Krebs-Henseleit (K-H) solution, as described by Ma and colleagues. ${ }^{24}$ Experiments were done by placing the cell dishes in an acrylic chamber which provided a controlled temperature $\left(37^{\circ} \mathrm{C}\right)$ and an atmosphere with the indicated amount of carbon dioxide $(5 \%)$, the balance being made up of nitrogen. For the hypoxia-reoxygenation experiments, the vein segments were exposed to $\mathrm{N}_{2}: \mathrm{CO}_{2}(95: 5)$ for 50 minutes followed by reoxygenation $\left(21 \% \mathrm{O}_{2}, 5 \% \mathrm{CO}_{2}, 74 \% \mathrm{~N}_{2}\right)$ for two hours at $37^{\circ} \mathrm{C}$.
Control vein segments were exposed to normoxia $\left(21 \% \mathrm{O}_{2}\right.$, $5 \% \mathrm{CO}_{2}, 74 \% \mathrm{~N}_{2}$ ) for the duration of the experiment (normoxic controls).

\section{Neutrophil isolation}

Neutrophils were isolated by Ficoll-Hypaque density gradient centrifugation, dextran sedimentation, and hypotonic lysis of erythrocytes. They were suspended in Hanks' balanced salt solution, free from phenol red, $\mathrm{Ca}^{2+}$, and $\mathrm{Mg}^{2+}$, and containing $0.25 \%$ bovine serum albumin. The final cell preparation had 98 (2)\% neutrophils (mean (SD)). The neutrophils were maintained on ice in Hanks' balanced salt solution at 1 to $5 \times 10^{6}$ cells/ml until used. Isolated neutrophils were more than $99 \%$ pure, as assessed by Wright's stained cytocentrifuge preparation, and more than $99 \%$ viable, as assessed by exclusion of trypan blue.

\section{Neutrophil CD1 1 b expression}

Neutrophil CDIlb expression was detected by indirect immunofluorescence and flow cytometry as previously described. ${ }^{25}$ In brief, $1 \mathrm{ml}$ of blood was drawn from the oxygenator into a heparinised syringe which was kept at room temperature to minimise the effects of cooling and rewarming on neutrophil integrin expression. Samples of blood in aliquots of $100 \mu \mathrm{l}$ were put in polypropylene tubes and stained with anti-CD1 lb monoclonal antibody CBL145 (Cymbus Biosciences, Hampshire, UK) as the primary reagent, and fluorescein isothiocyanate conjugated goat anti-mouse immunoglobulin (Cymbus Biosciences) as the secondary reagent. Flow cytometry was done on a FACScan (Becton Dickinson, Mountain View, California, USA). In the flow cytometry studies, the logarithmic mean fluorescence values obtained from the histograms were converted mathematically into a relative fluorescence value and expressed as per cent increase over the observed baseline values.

\section{Immunohistochemistry}

Immunostaining was used to investigate the extent of P-selectin expression in saphenous vein segments. Saphenous vein was dehydrated using graded acetone washes and embedded at $4^{\circ} \mathrm{C}$. Sections $6 \mu \mathrm{m}$ thick were cut and transferred to coated slides (Vectabond; Vector Laboratories, Burlingham, California, USA). The immunohistochemical localisation of P-selectin was accomplished using the avidinbiotin immunoperoxidase technique, as described by Chester and colleagues. ${ }^{26}$ Tissue sections were incubated with the primary antibody (Research Diagnostic, Flanders, New Jersey, USA, at 1:100 dilution) overnight at room temperature. A biotinylated IgG was used as the secondary antibody at a 1:200 dilution for one hour at room temperature. The avidin-biotin immunoperoxidase technique was used to detect biotinylated secondary antibody. Immunostaining negative controls included omission of the primary antibody or secondary antibody. P-selectin expression was defined according to Florens and colleagues, ${ }^{27}$ as the percentage of vein segments examined displaying brown reaction on $>50 \%$ of the circumference of its endothelium.

\section{Neutrophil adherence assay}

Neutrophils were fluorescently labelled with a hydrophobic fluorescent compound 3-3'- dioctadecyloxacarbocyanine perchlorate (DiI) (Fluka, Sigma-Aldrich, Milan, Italy), as previously described. ${ }^{28}$ Cells at a concentration of 4 to $8 \times 10^{6}$ cells $/ \mathrm{ml}$ were incubated with $50 \mu \mathrm{g} / \mathrm{ml}$ DiI in HAP buffer for 10 minutes at $0^{\circ} \mathrm{C}$, unbound dye was removed by three washes with HAP buffer, and labelled neutrophils were resuspended in medium 199 for the adhesion assay. After 10 minutes of preincubation of the vessel segments, autologous unstimulated DiI labelled neutrophils ( $10 \mu \mathrm{l}$ of $10^{6}$ cells $/ \mathrm{ml}$ ) were added and incubated for 20 minutes. Vessel segments were 
Table 1 Clinical deatils of the patients

\begin{tabular}{|c|c|c|c|}
\hline Variable & Group A & Group B & Group C \\
\hline Age (years) & $53.4(3.1)$ & $55(2.5)$ & $52.2(3.3)$ \\
\hline NYHA class & $2.2(0.1)$ & $2.3(0.3)$ & $2.1(0.7)$ \\
\hline \multicolumn{4}{|l|}{ Additional drugs } \\
\hline$\beta$ Blockers & 8 & 6 & 9 \\
\hline Calcium channel blockers & 13 & 11 & 15 \\
\hline Nitrates & 16 & 13 & 15 \\
\hline Total CPB time (min) & $96(7.7)$ & $103(6.5)$ & $101(9.2)$ \\
\hline Aortic cross clamp (min) & $48(5.4)$ & $52(4.8)$ & $46(6)$ \\
\hline Number of grafts & $2.2(0.3)$ & $2.3(0.2)$ & $2.2(0.4)$ \\
\hline Total cholesterol (mmol/I) & $5.11(0.16)$ & $6.94(0.17)$ & $4.44(0.20)$ \\
\hline HDL cholesterol (mmol/l) & 1.50 (0.09) & $1.30(0.11)$ & $1.14(0.11)$ \\
\hline Triglycerides & $1.93(0.14)$ & $2.01(0.21)$ & $1.89(0.24)$ \\
\hline \multicolumn{4}{|c|}{$\begin{array}{l}\text { Values are n or mean (SD). } \\
\text { Group A, hypercholesterolaemic, responsive to simvastatin; group B, hypercholesterolaemic, non-responsive } \\
\text { to simvastatin; group C, control. } \\
\text { CPB, cardiopulmonary bypass; HDL, high density lipoprotein; NYHA, New York Heart Association functiona } \\
\text { class. }\end{array}$} \\
\hline
\end{tabular}

then removed from culture dishes and dipped three or four times in fresh K-H solution. These vessel segments were then placed on a glass slide with the endothelial side up. The number of neutrophils adhering to the endothelial surface in five separate microscopic fields was counted manually on an inverted microscope equipped for fluorescence, using the filter IF355-550. Values of five replicates were averaged, and variations between replicates were $<10 \%$.

\section{Inhibition of NO release from saphenous vein endothelial cells}

We observed the effect of inhibiting basal NO release from the saphenous vein on neutrophil adherence to endothelium. NG-nitro-L-arginine methyl ester (L-NAME, $1 \mathrm{mmol} / \mathrm{l}$ ) was incubated with vessel segments for 30 minutes. These vessel segments were then transferred to fresh K-H solution that did not contain L-NAME. Stimulated autologous neutrophils were then incubated for another 20 minutes with these vessel segments. Neutrophil adhesion to the endothelium was evaluated as described earlier. In other experiments, the L-NAME vessel segments were coincubated with L-arginine $\left(10^{-2} \mathrm{~mol} / \mathrm{l}\right)$ or nitroprusside $(1 \mathrm{mmol} / \mathrm{l})$ to determine whether substrate or exogenous NO inhibited neutrophil adhesion to L-NAME treated vascular segments.

In a subset of experiments ( 10 for each group), the importance of neutrophil CDllb and endothelium P-selectin in determining neutrophil/endothelial adhesion was evaluated by treating neutrophils and saphenous vein endothelium with blocking monoclonal antibodies (mAb) to CDllb and P-selectin.

\section{Statistics}

Data are presented as mean (SEM). Raw data were analysed for normality of distribution. If not normally distributed, data were subjected to log transformation before analysis. We used $\chi^{2}$ tests to compare categorical variables or non-paired Student's $t$ tests as appropriate. Comparison between groups was made by using two way analysis of variance followed by the Bonferroni correction for $t$ test comparison. A probability value of $\mathrm{p}<0.05$ was considered significant.

\section{RESULTS}

\section{Patients}

Clinical and operative characteristics (shown in table 1) did not differ between the three groups. All patients had angina on effort and were receiving some combination of $\beta$ adrenergic blocking agents, nitrate vasodilators, and calcium channel blocking agents.

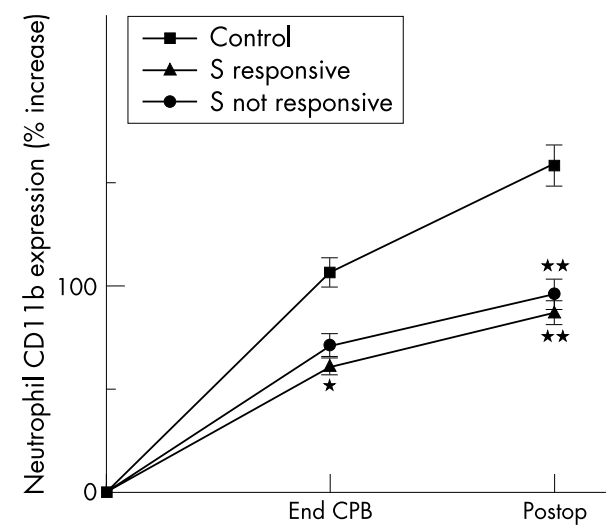

Figure 1 Expression of $C D 11 \mathrm{~b} \backslash C D 18$ on the surface of circulating neutrophils obtained at the end of cardiopulmonary bypass (End $\mathrm{CPB}$ ) and one hour postoperatively (Postop). ${ }^{*} p<0.05 v$ control; ${ }^{*} p<0.01 v$ control. S, simvastatin.

Total cholesterol concentrations were higher in patients not responsive to the simvastatin compared with both control and simvastatin responsive patients. Compared with the control groups, in the statin responsive group the preoperative lipid profile was beneficially affected by treatment, with significant lower concentrations of low density lipoprotein and a trend toward higher high density lipoprotein.

\section{Neutrophil CD18/CD11b expression}

Figure 1 shows neutrophil CDllb expression in control and simvastatin (S) treated patients. Values are expressed as a percentage of the baseline value (mean channel fluorescence: control, $\mathrm{n}=83$; simvastatin responsive, $\mathrm{n}=79$; simvastatin non-responsive, $\mathrm{n}=81$ ). In all patients, a significant increase in CDIlb expression was observed throughout the experimental period, with a peak at 60 minutes postoperatively. However, at this latter point, CDllb values from patients treated with simvastatin were significantly lower than in the control group.

\section{P-selectin expression on saphenous vein endothelial cells}

In normoxic vein segment from all groups, P-selectin staining was only weakly positive on the endothelial cells. We therefore investigated the effect of hypoxia $\left(95 \% \mathrm{~N}_{2}, 5 \% \mathrm{CO}_{2}\right)$ and reoxygenation $\left(95 \%\right.$ air, $5 \% \mathrm{CO}_{2}$ ) on the expression of P-selectin by saphenous vein endothelial cells. Figure 2 summarises the results and shows that, compared with normoxia, a period of hypoxia similar to that induced in the coronary endothelium 


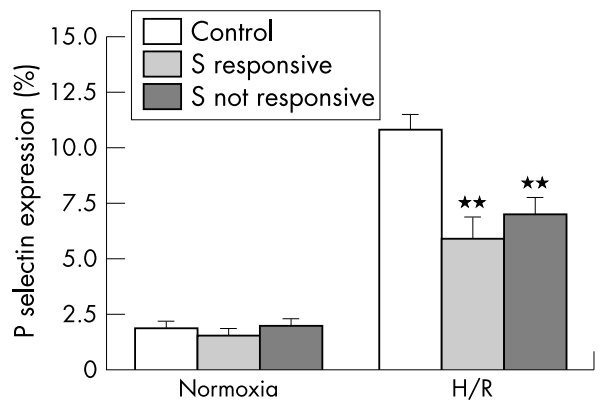

Figure 2 P-selectin expression of saphenous vein endothelium exposed to normoxia $\left(\mathrm{O}_{2} 21 \%\right)$ or 50 minutes of hypoxia $(H / R$, $\left.\mathrm{N}_{2}: \mathrm{CO}_{2}, 95: 5\right)$. The bars represent the mean number of positive staining vein segments/examined area. ${ }^{* *} \mathrm{p}<0.01 v$ control.

by aortic cross clamping resulted in a highly significant increase in endothelial P-selectin expression in vein segments from all groups (control: $\mathrm{p}<0.01 \quad v$ normoxia, mean difference $-8.9,95 \%$ confidence interval (CI) -11.5 to -6.3 ; simvastatin responsive: $\mathrm{p}<0.01 v$ normoxia, mean difference $-4.3,95 \%$ CI -6.9 to -1.8 ; simvastatin non-responsive: $\mathrm{p}<0.01 v$ normoxia, mean difference $-5.0,95 \%$ CI -7.9 to $-2.1)$. P-selectin expression was significantly reduced in vessel segments after simvastatin treatment, independently of its efficacy at lowering cholesterol (simvastatin responsive: $\mathrm{p}<0.01 v$ control, mean $4.8,95 \%$ CI 2.3 to 7.4 ; simvastatin non-responsive: $\mathrm{p}<0.01$, mean $3.8,95 \%$ CI 1.2 to 6.3 ).

\section{Neutrophil adhesion to saphenous vein endothelial cells}

Figure 3 shows the percentage of neutrophil adhesion to the endothelium of saphenous vein segments. Under baseline condition very few neutrophils bound to endothelium, and no significant difference was observed between the three groups. In contrast, in experiments with neutrophils obtained one hour postoperatively, adhesion and ruffle formation of neutrophils on normoxic endothelium were notably enhanced. However, on the basis of counts made before and after washing, the percentage of neutrophils adhering to the saphenous vein endothelium was significantly lower in the statin groups than in the control group (simvastatin responsive: $\mathrm{p}<0.01 v$ control, mean difference $7.5 \%, 95 \%$ CI 4.0 to 11.1; simvastatin non-responsive: $\mathrm{p}<0.01 v$ control, mean difference $6.7 \%, 95 \%$ CI 3.2 to 10.3$)$. When the neutrophils were incubated with vein segments after hypoxia/ reoxygenation, a greater number of adhering neutrophils was found compared with the normoxic vein segments. Again, the percentage of neutrophils adhering to the endothelium was lower in the statin groups than in the control group (simvastatin responsive: $\mathrm{p}<0.01 v$ control, mean difference $8.4 \%$, 95\% CI 4.9 to 12.0 ; simvastatin non-responsive: $\mathrm{p}<0.01$, mean difference $9.2 \%, 95 \%$ CI 5.7 to 12.8 ).

Figure 4 shows the results of a subset of experiments using hypoxic vein segments $(n=10)$, in which neutrophils obtained 60 minutes postoperatively were incubated with a blocking CDI lb antibody (clone 44a). The incubation of neutrophils with mAb 44a caused a decrease in neutrophil adhesion of $50 \%, 40 \%$, and $42 \%$ in the control, simvastatin responsive, and simvastatin non-responsive groups, respectively. The contribution of $\mathrm{P}$-selectin to ischaemia/reoxygenationenhanced adherence was determined by comparing exposing saphenous vein segments to a 1:200 dilution of blocking $\mathrm{P}$-selectin mAb 9E1. Figure 4 shows that incubation of the ischaemic/reoxygenated vein segment with mAb 9El further reduced the adhesion of mAb44 treated neutrophil to the vein endothelium (by $80 \%, 70 \%$, and 68\% in control, simvastatin responsive, and simvastatin non-responsive groups, respectively).

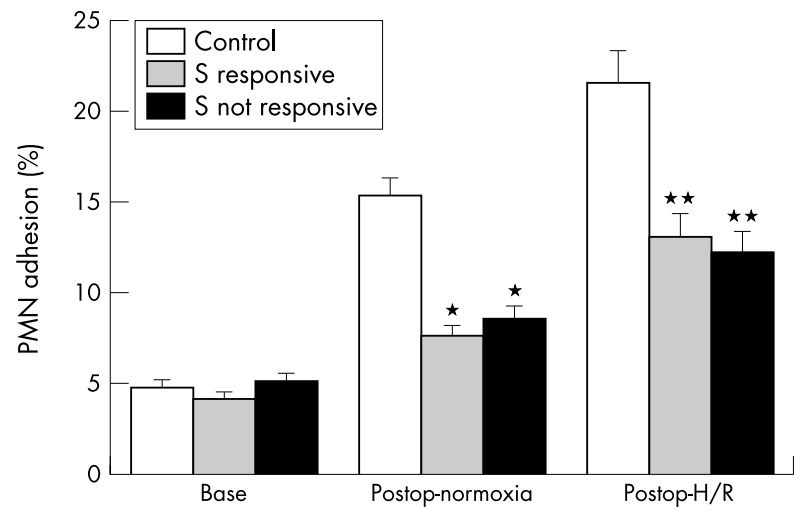

Figure 3 Graph showing the per cent adhesion of unstimulated leucocytes to the endothelium of saphenous vein under basal condition, and the adhesion of neutrophils drawn one hour postoperatively to either normoxic (Postop-normoxia) or hypoxic (Postop-H/R) vein endothelium. Height bar indicates mean values. ${ }^{*} p<0.05 v$ control; * $p<0.01 v$ control.

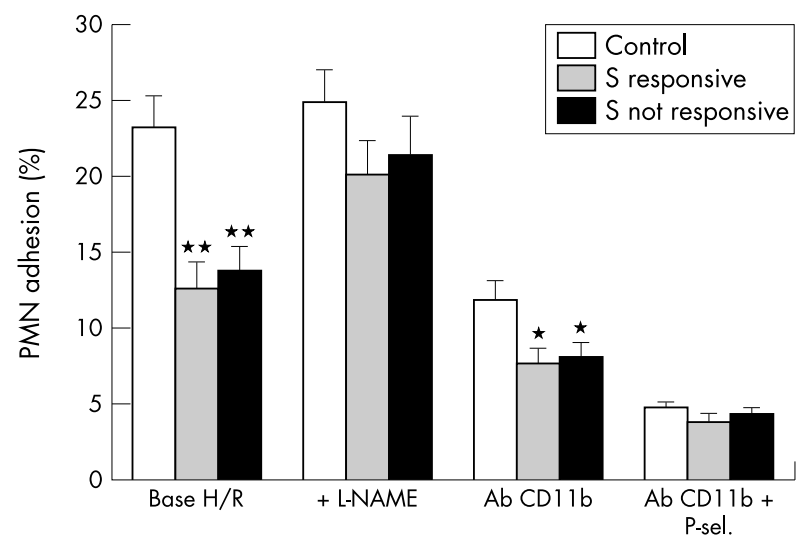

Figure 4 Graph showing activated leucocyte adhesion to hypoxic saphenous vein endothelium under basal condition (base $H / R$ ), after addition of L-NAME (+L-NAME), and after treatment with either blocking monoclonal antibody ( $\mathrm{mAb}$ ) to $\mathrm{CD} 111 \mathrm{~b}(\mathrm{Ab} C D 1 \mathrm{lb})$ or blocking mAbs to both CDI $1 \mathrm{~b}$ and P-selectin (Ab CDI 1b+P-sel). Height bar indicate mean values. ${ }^{*} p<0.05 v$ control; ${ }^{* *} p<0.01 v$ control.

In an attempt to relate the simvastatin modulation of neutrophil adherence to saphenous vein endothelium with enhanced basal NO production, we studied the effects of L-NAME added directly to the bath. Figure 4 summarises the results. Addition of L-NAME directly to the bath increased neutrophil adherence to the vein endothelium in the statin groups ( simvastatin responsive: $\mathrm{p}<0.05 v$ no L-NAME, mean difference -7.5 , 95\% CI -14.5 to -0.44 ; simvastatin nonresponsive: $\mathrm{p}<0.05 v$ no L-NAME, mean difference $-7.6,95 \%$ CI -15.0 to -0.2 ), with no significant difference observed between groups. Moreover, both the addition of L-arginine and supplementation of exogenous NO by nitroprusside significantly reduced the L-NAME induced neutrophil adhesion to vascular endothelium (data not shown). These results suggest that endogenous NO generated from vein endothelium acts as an inhibitor of neutrophil adherence.

\section{DISCUSSION}

Lipid lowering treatment with HMG-CoA reductase inhibitors has been shown to reduce cardiovascular morbidity and mortality in patients with and without cardiovascular disease. ${ }^{16172021}$ Datani and colleagues ${ }^{18}$ showed that preoperative statin treatment may be associated with a reduced incidence of post-CABG adverse cardiovascular outcomes. A 
reduced release of IL-6 during elective CABG after preoperative statin treatment has also been reported. ${ }^{29}$ Moreover, simvastatin treatment has been associated with improvement in coronary artery function together with decreased cytokine activation in patients after heart transplantation. ${ }^{30}$ Our results extend these conclusions and demonstrate that the benefits of statin treatment may also occur in the immediate postoperative period after CABG. In the present study, statin treatment was associated with lowered adhesion of activated neutrophils to vascular endothelium after hypoxia/reoxygenation, by a mechanism involving reduced expression of both neutrophil CDIlb and endothelial P-selectin. These endothelial protective effects are independent of the well known lipid lowering effects of statins, and occurred at clinically therapeutic doses in patients in whom statin treatment failed to lower the cholesterol concentrations. Our results are consistent with those of previous studies in which statins were found to inhibit both neutrophil activation and the process of P-selectin expression in the endothelial cell. ${ }^{1622}$ In a recent publication Florens and colleagues $^{27}$ failed to show an anti-inflammatory effect of acute pretreatment with atorvastatin $(40 \mathrm{mg}$ the evening before and $40 \mathrm{mg}$ on the morning of surgery). These differences in results could be explained by the acute setting of the study, with a time interval less than 24 hours between drug administration and the assay of the inflammatory response. In our study, treated patients had been on chronic treatment with statins for at least three months (median nine months, mean (SD) dose 26.4 (0.4) mg/day), and treatment of at least three months with statins was advocated in a previous study as being effective for improving the bioavailability of nitric oxide. ${ }^{31}$

\section{Modulation of neutrophil-endothelial interactions by statins}

The results of the present study confirm that cardiopulmonary bypass is associated with significant neutrophil activation, as evidenced by increased Macl expression. However, the most interesting observation was the significant attenuation of CDIlb neutrophil upregulation following treatment with statins, which in turn is responsible for a reduced neutrophil adhesion to the endothelial cells in both normoxic and ischaemia/reoxygenation experiments. Our findings are in agreement with those of Lefer and colleagues, ${ }^{32}$ who showed that simvastatin significantly inhibited the upregulation of CDllb expression on stimulated rat neutrophils by a mechanism not involving its cholesterol lowering effects. A similar effect has been described by Weber and associates for lovastatin, ${ }^{33}$ which decreases CDIlb expression and CDIlb dependent adhesion of monocytes to the endothelium in humans, independent of any cholesterol lowering effects.

The importance of surface CDl lb in mediating neutrophilendothelial adhesion has been demonstrated before. ${ }^{72} 132325$ In the present study, pretreatment with anti-Macl blocking antibodies significantly reduced neutrophil/endothelial adhesion in hypoxic experiments, although the number of neutrophils adhering to the endothelium was still increased, with higher values in control groups than in statin groups. This latter finding might certainly be explained by the increased expression of adhesion molecule P-selectin on the endothelial cell surface after hypoxia/reoxygenation, which would account for the rolling and the early adhesion of neutrophils. In fact, incubation of endothelial cell with monoclonal blocking antibodies against P-selectin almost completely abolished neutrophil adhesion.

P-selectin is an endothelial adhesion molecule found constitutively in a preformed state in the $\alpha$ granules of platelets and in the Wiebel-Palade bodies of endothelial cells. It is rapidly translocated to the cell surface after stimulation with inflammatory mediators, allowing rolling and adhesion of activated neutrophils to the endothelium. ${ }^{34}$ This latter phenomenon is crucial in mediating leucocyte adhesion to platelets and endothelium after ischaemia/reoxygenation.

Pinsky and colleagues ${ }^{4}$ demonstrated P-selectin exocytosis on coronary endothelial cells of patients undergoing routine cardiac surgery under hypothermic cardioplegic arrest. In our study, we confirmed the observation that a relatively brief period of hypoxia followed by reoxygenation elicits a significant increase in P-selectin at the endothelial surface of saphenous vein..$^{35}$ However, vein segments from a patient under statin treatment expressed significantly less P-selectin on the endothelial surface after 50 minutes of hypoxia followed by reperfusion, with a beneficial effect on leucocyteendothelial interactions compared with controls. This effect was not related to the cholesterol lowering effect of simvastatin, as it was also manifested in group B patients who did not benefit from simvastatin treatment in terms of reduced cholesterol. Our results are in agreement with those of Pruefer and colleagues, ${ }^{22}$ who showed a significant attenuation in the upregulation of P-selectin on the mesenteric endothelium in an acute inflammatory state after treatment with simvastatin.

In conclusion, in experiments using either blood vessels or neutrophils isolated from statin treated patients, adherence was significantly attenuated. Thus simvastatin exerts an antiadherence effect acting both on neutrophils and on the endothelium.

\section{Statin treatment improves endothelial function after ischaemia/reperfusion}

Endothelial dysfunction has long been shown to be a critical early component of organ injury following myocardial ischaemia and reperfusion during coronary surgery under cardiopulmonary bypass, ${ }^{1-3}$ and has led to the investigation of strategies to prevent its occurrence. Moreover, cardiopulmonary bypass itself causes local injury secondary to an acute inflammatory response that involves tissue infiltration by activated neutrophils and platelets. ${ }^{5-9}$ The mechanism of endothelial dysfunction and reduced NO bioavailability in ischaemia/ reperfusion injury is still being debated. Data from studies in animals suggest that there might either be reduced substrate for NO synthesis ${ }^{10}$ or increased NO inactivation by neutrophil released superoxide. ${ }^{36}$ Recent results suggest that the cardioprotective effects of statins can be at least partially explained by the enhancement of endothelial NO synthase (eNOS) expression, which therefore overcomes the hypoxia mediated inhibition of NO synthesis activity. ${ }^{19-21}$

Laufs and colleagues ${ }^{37}$ have shown that simvastatin and lovastatin improve the stability of the mRNA for eNOS and increase the half life of the mRNA for eNOS from 13 to 38 hours, with a consequent enhanced generation of NO from the endothelium. Wassmann and colleagues showed that 30 days of treatment with atorvastatin in normocholesterolaemic, spontaneously hypertensive rats caused an upregulation of eNOS mRNA expression (138 (7)\% of control) and an enhanced eNOS activity in the vessel wall $(209(46) \%$ of control). ${ }^{38}$ Moreover, treatment with atorvastatin caused a significant reduction in systolic blood pressure and a profound improvement in endothelial dysfunction mediated by a reduction in free radical release in the vasculature. Upregulation of endothelial NO synthesis, as well as inhibition of hypoxia mediated inhibition of NOS activity, has been observed with simvastatin and lovastatin in mice subjected to cerebral ischaemia-reperfusion. These effects were dependent on enhanced NO formation because they failed to ameliorate the high leucocyte rolling and adherence in eNOS deficient mice. ${ }^{39}$

The results of the present study are in accordance with these past reports and show that the downregulation of both P-selectin expression on endothelial cells and CD18 on stimulated neutrophils is mediated by enhanced NO synthesis. In our experiments, the addition of L-NAME, an inhibitor of NO 
synthase, in the culture media of vein segments almost completely abolished the statin effect on neutrophil-endothelial adhesion. In the same fashion, when L-NAME was added to neutrophils withdrawn at the end of cardiopulmonary bypass, the number of neutrophils adhering to non-stimulated endothelial cells did not differ between the control group and the treatment groups.

\section{Limitations of the study}

In our study, we tested neutrophil-endothelial adhesion by using segments of saphenous vein subjected to a brief period of controlled hypoxia/reoxygenation. In the clinical setting of coronary artery bypass, ischaemia/reperfusion injury mainly involves the coronary endothelium, in which the $\mathrm{Po}_{2}$ declines to $<20$ torr. $^{40}$ Nevertheless, we can only speculate that the observation made with a saphenous vein model could be extrapolated to the coronary endothelium. Furthermore, ischaemia/reperfusion is not the only cause of the endothelial dysfunction which occurs during cardiopulmonary bypass. After operation there are several potential stimuli for the expression of adhesion molecules in veins. These include the action of the cytokines released as a result of cardiopulmonary bypass, and increased stretch in the vessel wall because of mechanical distension and exposure to arterial pressure. In the controlled setting of our experiment, we could not consider the influence on the endothelial cells of cytokine release, mechanical wall stress, and the action of activated neutrophils themselves.

\section{Conclusions}

Although further studies are advisable to clarify the exact mechanism of action, we can affirm on the basis of our data that pretreatment with simvastatin significantly reduces neutrophil adhesion to the venous endothelium, by an NO mediated mechanism, in patients undergoing coronary artery bypass grafting with cardiopulmonary bypass, irrespective of its efficacy at lowering cholesterol concentrations.

\section{Authors' affiliations}

M Chello, G Patti, A D'Ambrosio, M Cortez Morichetti, G Di

Sciascio, E Covino Interdisciplinary Centre for Biomedical Research $(\mathrm{CIR})$, Department of Cardiovascular Sciences, University Campus Bio-Medico di Roma, Rome, Italy

E Mastroroberto, Department of Clinical and Experimental Medicine,

University of Catanzaro, Catanzaro, Italy

\section{REFERENCES}

1 Verrier ED, Boyle EM. Endothelial cell injury in cardiovascular surgery: an overview. Ann Thorac Surg 1997;64:S2-8.

2 Asimakopoulos G, Taylor KM. Effects of cardiopulmonary bypass on leukocyte and endothelial adhesion molecules. Ann Thorac Surg 1998;66:2135-44

3 Lefer AM, Tsao PS, Lefer DJ, et al. Role of endothelial dysfunction in the pathogenesis of reperfusion injury after myocardial ischemia. FASEB J 1991;5:2029-34.

4 Pinsky DJ, Naka Y, Liao $\mathrm{H}$, et al. Hypoxia-induced exocytosis of endothelial cell Weibel-Palade bodies. J Clin Invest 1996;97:493-500.

5 Asimakopoulos G. Systemic inflammation and cardiac surgery: an update. Perfusion 2001;16:353-60.

6 Treacher DF, Sabbato M, Brown KA, et al. The effects of leucodepletion in patients who develop the systemic inflammatory response syndrome following cardiopulmonary bypass. Perfusion $2001 ; 16$ (suppl):67-73.

7 Kawahito K, Kobayashi E, Ohmori M, et al. Enhanced responsiveness of circulatory neutrophils after cardiopulmonary bypass: increased aggregability and superoxide producing capacity. Artif Organs 2000;24:37-42.

8 Menaschè $\mathbf{P}$. The systemic factor: the comparative roles of cardiopulmonary bypass and off pump surgery in the genesis of patient injury during and following cardiac surgery. Ann Thorac Surg 2001;72:S2260-6.

9 Hayashi Y, Saewa Y, Fukuyama N, et al. Inducible nitric oxide production is an adaptation to cardiopulmonary bypass-induced inflammatory response. Ann Thorac Surg 2001;72:149-55.

10 Engelman DT, Watanabe M, Engelman RM, et al. Constitutive nitric oxide release is impaired after ischemia and reperfusion. J Thorac Cardiovasc Surg 1995;110:1047-53.
11 Kubes P, Suzuki M, Granger D. Nitric oxide: an endogenous modulator of leukocyte adhesion. Proc Natl Acad Sci USA 1991;88:4651-5.

12 Krieglstein CF, Granger DN. Adhesion molecules and their role in vascular disease. Am J Hypertens 2001;14:44-54S.

13 Gauthier TW, Davenpeck KL, Lefer AM. Nitric oxide attenuates leukocyte endothelial interaction via P-selectin in splanchnic ischemia-reperfusion. Am J Physiol 1994;26:G562-8.

14 Oyama J, Shimokawa H, Momii H, et al. Role of nitric oxide and peroxynitrite in the cytokine induced sustained myocardial dysfunction in dogs in vivo. J Clin Invest 1998;1 10:2207-14.

15 Worrall NK, Chang K, Lejeunne WS, et al. TNF-alpha causes reversible in vivo systemic vascular barrier dysfunction via NO-dependent and independent mechanism. Am J Physiol 1997;273:H2565-74.

16 Murphy RT, Foley JB, Mulvihill N, et al. Impact of pre-existing statin use on adhesion molecule expression in patients presenting with acute coronary syndromes. Am J Cardiol 2001;87:446-8.

17 Sacks FM, Pfeffer MA, Moye LA, et al. The effect of pravastatin on coronary events after myocardial infarction in patients with average cholesterol levels. N Engl J Med 1996;335:1001-9.

18 Datani MI, Elnicki M, Jain AC, et al. Effect of preoperative statin therapy and cardiac outcome after coronary artery bypass grafting. Am J Cardiol 2000;86: 1 128-30.

19 Lefer AM, Scalia R, Lefer DJ. Vascular effects of HMG CoA-reductase inhibitors (statins) unrelated to cholesterol lowering: new concept for cardiovascular disease. Cardiovasc Res 2001;49:281-7.

20 Miller SJ. Emerging mechanisms for secondary cardioprotective effects of statins. Cardiovasc Res 2001:52:5-7.

21 Takemoto $M$, Liao JK. Pleiotropic effects of 3-hydroxy-3-methylglutaryl coenzyme a reductase inhibitors. Arterioscler Thromb Vasc Biol 2001;21:1712-19

22 Pruefer D, Scaliia R, Lefer AM. Simvastatin inhibits leukocyte-endothelial cell interactions and protects against inflammatory processes in normocholesterolemic rats. Arterioscler Thromb Vasc Biol 1999; 19:2894-900.

23 Chello M, Mastroroberto P, Malta E, et al. Inhibition by dipyridamole of neutrophil adhesion to vascular endothelium during coronary bypass surgery. Ann Thorac Surg 1999;67:1277-82.

24 Ma X, Weyrich AS, Lefer DJ. Diminished basal nitric oxide release after myocardial ischemia and reperfusion promotes neutrophil adherence to coronary endothelium. Circ Res 1993;77:403-12.

25 Chello M, Mastroroberto P, Marchese AR, et al. Nitric oxide inhibits neutrophil adhesion during experimental extracorporeal circulation. Anesthesiology 1998;89:443-8.

26 Chester AH, Morrison KJM, Yacoub MH. Expression of vascular adhesion molecules in saphenous vein coronary bypass. Ann Thorac Surg 1998;65: 1685-9.

27 Florens E, Salvi S, Peynet J, et al. Can statins reduce inflammatory response to cardiopulmonary bypass? A clinical study. J Card Surg $2001 ; 16: 232-9$.

28 Chello M, Mastroroberto P, Perticone F, et al. Nitric oxide modulation of neutrophil-endothelium interaction: difference between arterial and venous coronary bypass grafts. J Am Coll Cardiol 1998;31:823-6.

29 Brull JD, Sanders J, Rumley A, et al. Statin therapy and the acute inflammatory response after coronary artery bypass grafting. Am J Cardiol 2001:88:431-3.

30 Weis M, Pehlivanli S, Meiser BM, et al. Simvastatin treatment is associated with improvement in coronary endothelium function and decreased cytokine activation in patients after heart transplantation. J Am Coll Cardiol 2001;38:814-18.

31 O'Driscoll G, Green D, Taylor RR. Simvastatin, an HMG-coenzyme A reductase inhibitor, improves endothelial function within 1 month. Circulation 1997;95:1126-31

32 Lefer AM, Campbell B, Shin YK, et al. Simvastatin preserves the ischemic-reperfused myocardium in normocholesterolemic rat hearts. Circulation 1999;100:178-84.

33 Weber C, Erl W, Weber KSC, et al. HMG-CoA reductase inhibitors decrease CD $11 \mathrm{~b}$ expression and CD $11 \mathrm{~b}$-dependent adhesion of monocytes to endothelium and reduce increased adhesiveness of monocytes isolated from patients with hypercholesterolemia. J Am Coll Cardiol 1997:30:1212-17.

34 Patel KD, Zzimmerman GA, Prescott SM, et al. Oxygen radicals induce human endothelial cells to express MP-140 and bind neutrophils. J Cell Biol 1991;112:749-59.

35 Closse $C$, Seigneur M, Renard M, et al. Influence of hypoxia and hypoxia-reoxygenation on endothelial P-selectin expression. Thromb Res 1997:85: 159-64.

36 Kurose I, Anderson DC, Miyasaka M, et al. Molecular determinants of reperfusion-induced leukocyte adhesion and vascular protein leakage. Circ Res 1994;74:336-43.

37 Laufs U, La fata V, Liao JK. Inhibition of 3-hydroxyy-3-methylglutaryl (HMG)-CoA reductase blocks hypoxia-mediated down regulation of endothelial nitric oxide synthase. J Biol Chem 1997;272:31725-9.

38 Wassmann S, Laufs U, Baumerr AT, et al. HMG-CoA reductase inhibitors improve endothelial dysfunction in normocholesterolemic hypertension via reduced production of reactive oxygen species. Hypertension 2001;37:1450-7.

39 Endres $M$, Laufs U, Huamg Z, et al. Stroke protection by 3-hydroxy-3-methylglutaryl (HMG)-CoA reductase inhibitors mediated by endothelial nitric oxide synthase. Proc Natl Acad Sci USA 1998;95:8880-5

40 Pinsky DJ, Oz MC, Liao S, et al. Restoration of the cAMP second messenger pathway enhances cardiac preservation for transplantation in a heterotopic rat model. J Clin Invest 1993;92:2994-3002. 